\title{
Imaging of synapses in 3D with non-destructive synchrotron X-ray ptychography
}

\author{
C. Bosch¹, A. Diaz², A. Pacureanu³ ${ }^{3}$ M. Holler², E. Müller ${ }^{4}$, A. Schaefer ${ }^{1}$ \\ ${ }^{1}$ The Francis Crick Institute, London, UK, ${ }^{2} c S A X S$ beamline, Paul Scherrer Institut, Villigen, Switzerland, ${ }^{3} I D 16 A$ beamline, ESRF, \\ Grenoble, France, ${ }^{4}$ Electron Microscopy Facility, Paul Scherrer Institut, Villigen, Switzerland
}

Email of communicating carles.bosch@crick.ac.uk

Wiring diagrams of neural circuits are of central importance in delineating mechanisms of computation in the brain (1). Hereby, the individual parts of neurons - axons, dendrites and synapses - need to be densely identified in 3-dimensional volumes of neuronal tissue. This is typically achieved by volume electron microscopy (2), which requires ultrathin physical sectioning or ablation, using high precision slicing techniques or ion beams, either before or during the image acquisition process (3-6). Here, we demonstrate that cryogenic X-ray ptychographic tomography (7-9), a coherent diffractive X-ray imaging technique, can acquire 3-dimensional images of metal-stained mouse neuronal tissue with sufficient resolution to densely resolve axon bundles, boutons, dendrites and synapses without physical sectioning. We show that the tissue volume can be subsequently imaged in 3D using high-resolution, focussed ion beam-scanning electron microscopy (FIB-SEM). This suggests that metal-stained neuronal tissue can be highly radiation-stable. Using FIB-SEM as ground truth, we could show that X-ray ptychography reliably resolves $60 \%$ of the synaptic contacts in the mouse olfactory bulb external plexiform layer with an $80 \%$ precision. Ongoing improvements in synchrotron, X-ray and detector technologies $(8,10,11)$ as well as further optimization of sample preparation and staining procedures $(12,13)$ could lead to substantial improvements in acquisition speed. Combined with laminography (14) and nano-holotomography $(15,16)$ it could allow for nondestructive x-ray imaging of synapses and neural circuits in increasingly larger volumes.

1. A. Litwin-Kumar, S. C. Turaga, Constraining computational models using electron microscopy wiring diagrams. Current opinion in neurobiology 58, 94-100 (2019).

2. J. Kornfeld, W. Denk, Progress and remaining challenges in high-throughput volume electron microscopy. Current opinion in neurobiology 50, 261-267 (2018).

3. W. Denk, H. Horstmann, Serial block-face scanning electron microscopy to reconstruct three-dimensional tissue nanostructure. PLoS biology 2, e329 (2004).

4. K. J. Hayworth et al., Gas cluster ion beam SEM for imaging of large tissue samples with 10 nm isotropic resolution. Nature methods 17, 68-71 (2020).

5. C. S. Xu et al., Enhanced FIB-SEM systems for large-volume 3D imaging. Elife 6, (2017).

6. N. Kasthuri et al., Saturated Reconstruction of a Volume of Neocortex. Cell 162, 648-661 (2015).

7. P. Thibault et al., High-resolution scanning x-ray diffraction microscopy. Science 321, 379-382 (2008).

8. M. Holler et al., High-resolution non-destructive three-dimensional imaging of integrated circuits. Nature 543, 402-406 (2017).

9. H. T. Tran et al., Alterations in Sub-Axonal Architecture Between Normal Aging and Parkinson's Diseased Human Brains Using LabelFree Cryogenic X-ray Nanotomography. Front Neurosci 14, 570019 (2020).

10. M. Eriksson et al., Some small-emittance light-source lattices with multi-bend achromats. Nuclear Instruments and Methods in Physics Research Section A: Accelerators, Spectrometers, Detectors and Associated Equipment 587, 221-226 (2008).

11. J. H. Jungmann-Smith et al., Radiation hardness assessment of the charge-integrating hybrid pixel detector JUNGFRAU 1.0 for photon science. Rev Sci Instrum 86, 123110 (2015).

12. Y. Hua, P. Laserstein, M. Helmstaedter, Large-volume en-bloc staining for electron microscopy-based connectomics. Nature communications 6, 7923 (2015).

13. S. Mikula, W. Denk, High-resolution whole-brain staining for electron microscopic circuit reconstruction. Nature methods 12, 541-546 (2015).

14. M. Holler et al., Three-dimensional imaging of integrated circuits with macro- to nanoscale zoom. Nature Electronics 2, 464-470 (2019).

15. P. Cloetens, J. Baruchel, Holotomography: Quantitative phase tomography with micrometer resolution using hard synchrotron radiation $\mathrm{x}$ rays. Applied Physics Letters 75, (1999).

16. A. T. Kuan et al., Dense neuronal reconstruction through X-ray holographic nano-tomography. Nat Neurosci 23, 1637-1643 (2020).

\section{Keywords: synapse; ptychography; neuroscience}

We acknowledge the Paul Scherrer Institut, Villigen, Switzerland for provision of synchrotron radiation beamtime at the cSAXS beamline X12SA of the SLS under the proposals 20190654 and 20200783. 